\title{
A Suspected Ovarian Cancer in a Postmenopausal Woman That Turned Out to Be a Broad Ligament Leiomyoma with Extensive Hydropic Degeneration
}

\author{
Thoshya Polepalle, Navindi Hasara Fernandopulle* and Jaydip Raut
}

Gloucestershire Royal Hospital, Gloucester, UK

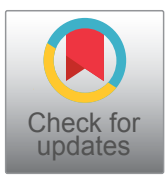

*Corresponding author: Navindi Hasara Fernandopulle, Gloucestershire Royal Hospital, Gloucester, Flat 48, Robinswood House, Gloucester, UK, Tel: +447592517767

\section{Summary}

Leiomyomas affect $30 \%$ of all women of reproductive age. They can be of uterine or extrauterine origin. Broad ligament leiomyomas are a type of extrauterine leiomyomas, its incidence comprising less than $1 \%$. It can undergo degenerative changes. Extensive hydropic degeneration is a rare occurrence and is commonly reported in association with pregnancy. It is a close mimic of malignancy due to its rapid growth and atypical imaging appearances 3 which can lead to diagnostic challenges.

We present a case of a 63-year-old female who was pre-operatively suspected to have an ovarian malignancy but turned out to be a benign looking broad ligament mass, cystic in nature on laparotomy. Total abdominal hysterectomy with bilateral salingo-oopherectomy was performed along with removal of mass and it was confirmed to be a leiomyoma with extensive hydropic degeneration on histological examination.

\section{Background}

Leiomyomas affect $30 \%$ of all women of reproductive age. They can be of uterine or extrauterine origin. There are several different types of extrauterine leiomyomas, including cervical leiomyomas, vaginal leiomyomas, broad ligament leiomyomas, disseminated peritoneal leiomyomatosis, intravenous leiomyomatosis of the pelvis, benign metastasising leiomyoma in the pelvis and retroperitoneal pelvic leiomyoma [1]. Presenting signs and symptoms depend on their size and location and include uterine bleeding, pain, palpable abdominal mass and infertility. The incidence of broad-ligament leiomyoma is less than $1 \%$ [2].

These benign tumours commonly undergo degenerative changes. The types of degeneration are hyaline, cystic (extreme form of hydropic degeneration), myxoid and red degeneration. Out of these, extensive hydropic degeneration is a rare occurrence and is commonly reported in association with pregnancy. It is a close mimic of malignancy due to its rapid growth and atypical imaging appearances [3] which can lead to diagnostic challenges.

Hydropic degeneration is characterized by accumulation of fluid with associated collagen deposition presenting with various patterns $[4,5]$. Leiomyomas with hydropic change do not have thick smooth muscle cells fascicles but a delicate filigree pattern with fluid (p.1508-1511) [6]. Hydropic changes lead to the formation of cystic cavities and this can cause dilemma in diagnosis as they mimic ovarian masses.

We present a 63-year-old female who was preoperatively suspected to have an ovarian mass but turned out to be a broad ligament cystic mass on laparotomy which was confirmed as a leiomyoma with extensive hydropic degeneration on histological examination. This is an important case as extensive hydropic degeneration in uterine leiomyoma is a rare occurrence and is commonly reported in association 

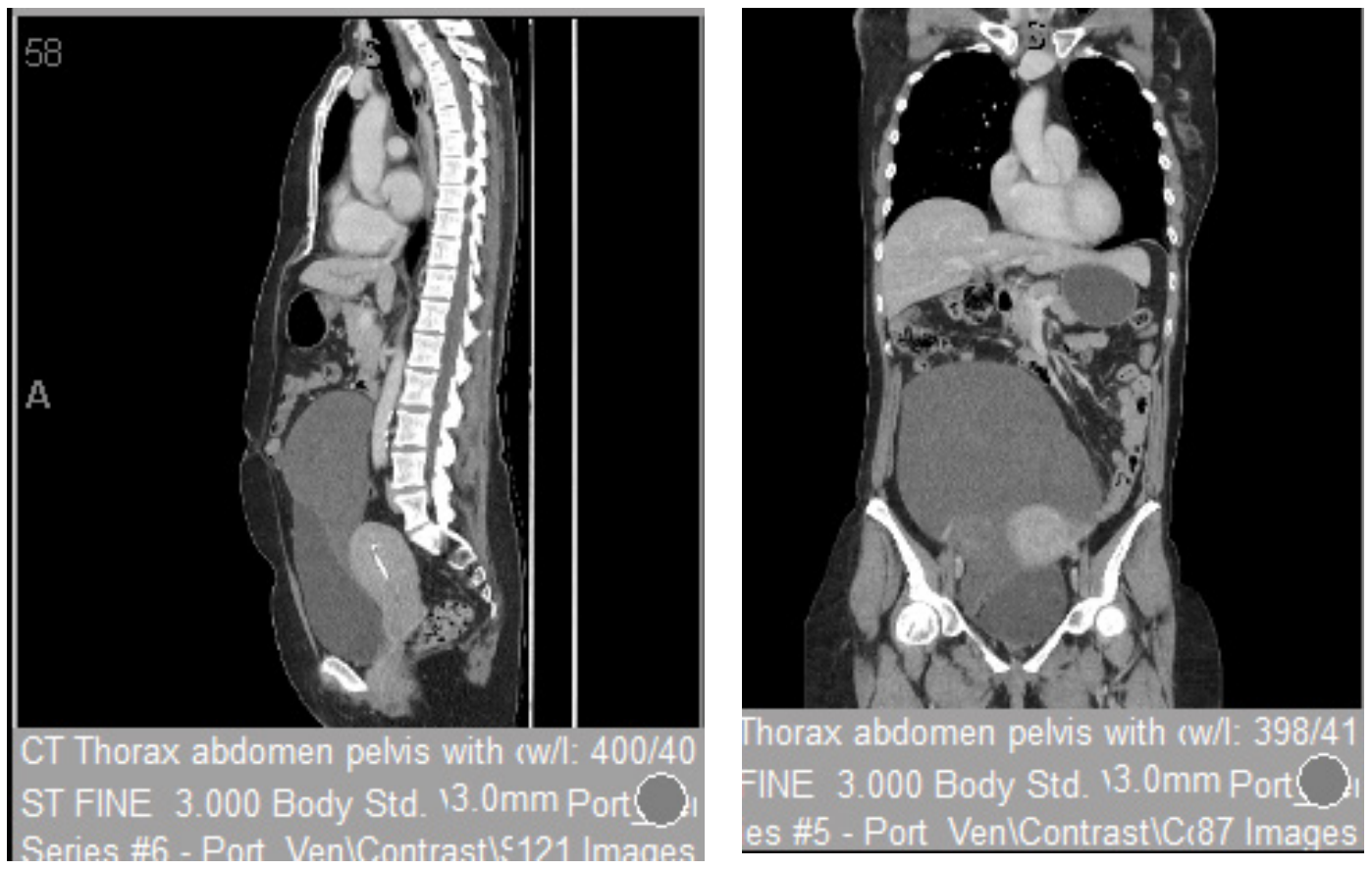

Figure 1: CT thorax abdomen and pelvis revealed a large complex cystic mass filling the central and right pelvis and extending to above the level of umbilicus.

with pregnancy. It usually occurs in reproductiveaged women and its occurrence in their sixties has not been reported to our knowledge. It is a close mimic of malignancy due to rapid growth. This case report draws attention to the possibility of it occurring in a female of non child bearing age. It is worth considering leiomyoma with hydropic degeneration as a differential diagnosis whilst dealing with patients presenting with cystic pelvic masses irrespective of their age group to avoid unnecessary extensive surgery.

\section{Case Presentation}

A 63-year-old female presented to our department with post-menopausal bleeding and abdominal distension. CA-125 was $29 \mathrm{U} / \mathrm{mL}$. Endometrial biopsy revealed no hyperplasia or malignancy.

\section{Investigations}

Imaging studies were performed. A CT thorax abdomen and pelvis revealed a large complex cystic mass filling the central and right pelvis and extending to above the level of umbilicus. It was suspected to be of ovarian origin due to the age of the patient, location, size and cystic nature of the mass (Figure 1).

The case was discussed in a multi-disciplinary team (MDT) meeting and a decision was made to perform laparotomy and total abdominal hysterectomy and bilateral salpingo-oophorectomy. Based on frozen section biopsy report, a decision would be made whether to proceed with an appendicectomy and lymph node dissection.

\section{Treatment}

She underwent prompt surgery as recommended by the MDT meeting. Intraoperatively, the uterus and fallopian tubes appeared normal, both ovaries were atrophic. No obvious metastases were noted. A large cystic mass in the right broad ligament was noted which extended superiorly until the umbilicus (24 weeks' size), inferiorly up to the transverse cervical ligament. Total abdominal hysterectomy with bilateral salpingooopherectomy with removal of mass was performed. Macro-anatomy strongly suggested the benign nature and thus we did not perform further surgery including lymph node dissection.

\section{Outcome and Follow-Up}

On Postoperative histopathological examination of the Broad ligament mass (weight: $1148.2 \mathrm{~g}$, Size: $160 \times$ $130 \times 85 \mathrm{~mm}$ ) the cut surface was solid, cystic with areas of mucin and firm, pale areas. No areas of necrosis or haemorrhage were apparent. Sections from the tumour showed a well circumscribed mass with alternating zones of cellularity. The hypo-cellular zones had an oedematous appearance with cystic degeneration. The solid areas contained a prominent vascular component associated with spindled to epithelioid cells arranged around them in nests or in long fascicles. There were numerous mast cells scattered throughout the lesion. There were no areas of necrosis or haemorrhage, and mitoses were rare. Immunohistochemistry shows that the cells are strongly positive for desmin and SMA, negative for $\mathrm{CD} 34$, and have variable ER positivity. They were negative for $A E 1 / 3$ and $S 100$, although mast cells show positivity for these markers. These features are in keeping with a leiomyoma with extensive hydropic degeneration (Figure 2 and Figure 3 ).

Post-operatively, the patient recovered well and was 


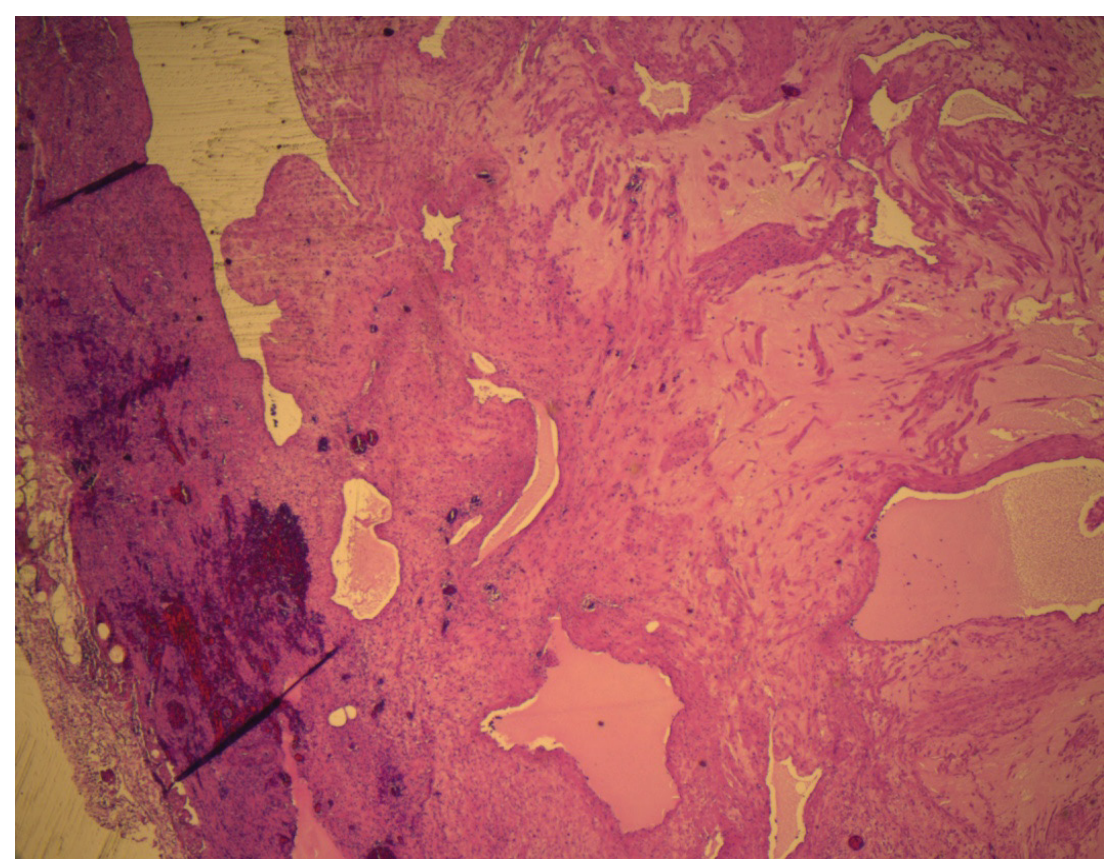

Figure 2: Histopathological image of the Broad ligament mass.

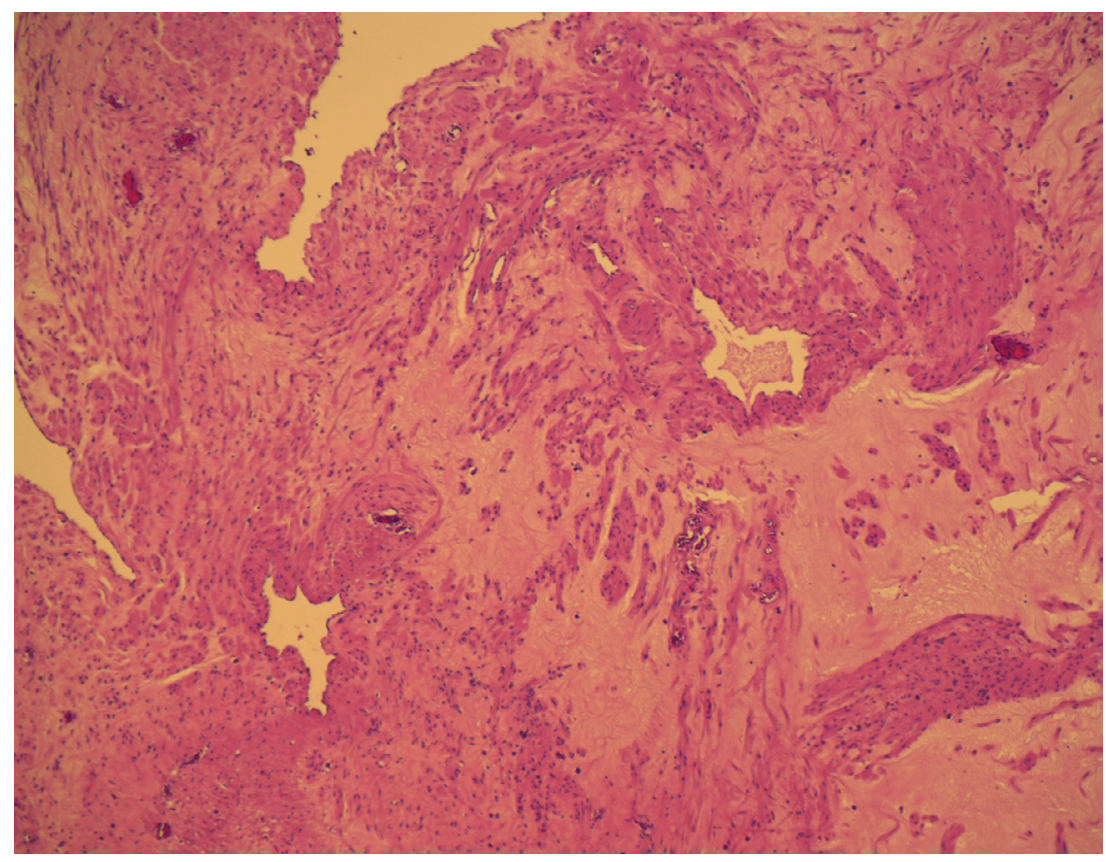

Figure 3: Histopathological image of the Broad ligament mass.

sent home on day 3. The patient was informed of the histology results. No further follow up was arranged because of the benign nature of the mass.

\section{Discussion}

Uterine leiomyomas are benign tumours arising from the smooth muscle cells of the uterus enclosed by a pseudocapsule [7,8]. As the tumours grow in size, an imbalance occurs between oxygen demand by the tumour cells and the available supply leading to focal ischaemia causing a foci of degeneration [9]. Hyaline, myxoid, red and cystic are the various types of degenerations described so far. Malignant transformation is observed in $0.5 \%$ of leiomyomas [7].
Cystic degeneration is thought to represent around $4 \%$ of the types of degeneration [2]. Cystic degeneration of leiomyomas is an extreme form of hydropic degeneration and can mimic other abdominopelvic masses like ovarian tumours $[10,11]$, endometriomas, abscess, adenomyosis [8], and uterine sarcomas. On ultrasound, fibroids with degenerative changes are either seen as hypoechoic or heterogenous uterine masses with cystic areas. Fibroids with this type of degeneration may show cystic areas with portions of a high T2 signal on MRI [12].

Complex cystic adnexal masses can be distinguished from uterine masses in most cases but there are 
exceptions which include pedunculated leiomyoma with cystic degeneration, cystic adenomyosis and a unicornuate uterus with a rudimentary obstructed horn [13]. In the case presented, it was appropriate to suspect an ovarian malignancy as the patient is of 63 years of age and the mass is of complex nature. Although the CA125 was well within normal limits, it is known that the positive predictive value of CA 125 in women with an adnexal mass is $35 \%$ to $91 \%$, and the negative predictive value ranges between $67 \%$ and $90 \%$ [14]. Imaging beyond ultrasound also could not determine the origin of mass in this case. Therefore, it was discussed in MDT meeting and a decision for laparotomy and frozen section was made. Intra-operatively, it was quite clear that the mass is of benign nature and so frozen section and pelvic lymph node sampling was not performed.

A high index of suspicion is needed in assessing complex adnexal masses and degenerating fibroids have to be included in the differential diagnosis. Also, it is of utmost importance to avoid extensive surgeries in patients with benign pathology and decision making intra-operatively based on clinical findings is very important.

\section{Learning Points/Take Home Messages}

- Cystic degeneration of leiomyomas is an extreme form of hydropic degeneration.

- Extensive hydropic changes can occur rarely in females of non child bearing age too.

- Findings intra-operatively might be different from what we expect and the extent of surgery needs to be optimised accordingly.

\section{References}

1. Fasih N, Prasad Shanbhogue AK, Macdonald DB, FraserHill MA, Papadatos D, et al. (2008) Leiomyomas beyond the uterus: Unusual locations, rare manifestations. Radiographics 28: 1931-1948.
2. Mallick D, Saha M, Chakrabarti S, Chakraborty J (2014) Leiomyoma of broad ligament mimicking ovarian malignancy-report of a unique case. Kathmandu Univ Med J (KUMJ) 12: 219-221.

3. Patil AR, Nandikoor S, Padilu R (2018) Hydropic degeneration of leiomyoma in nongravid uterus: The "split fiber" sign on magnetic resonance imaging. Indian J Radiol Imaging 28: 182.

4. Clement PB, Young RH, Scully RE (1992) Diffuse, perinodular, and other patterns of hydropic degeneration within and adjacent to uterine leiomyomas. Problems in differential diagnosis. Am J Surg Pathol 16: 26-32.

5. Coad JE, Sulaiman RA, Das K, Staley N (1997) Perinodular hydropic degeneration of a uterine leiomyoma: A diagnostic challenge. Hum Pathol 28: 249-251.

6. Rosai J (2011) Rosai and Ackerman's surgical pathology e-book. Elsevier Health Sciences.

7. Lev-Toaff AS, Coleman BG, Arger PH, Mintz MC, Arenson $\mathrm{RL}$, et al. (1987) Leiomyomas in pregnancy: Sonographic study. Radiology 164: 375-380.

8. Murase E, Siegelman ES, Outwater EK, Perez-Jaffe LA, Tureck RW (1999) Uterine leiomyomas: Histopathologic features, MR imaging findings, differential diagnosis, and treatment. Radiographics 19: 1179-1197.

9. Protopapas A, Milingos S, Markaki S, Loutradis D, Haidopoulos D, et al. (2008) Cystic uterine tumors. Gynecol Obstet Invest 65: 275-280.

10. Sherer DM, Maitland CY, Levine NF, Eisenberg C, Abulafia O (2000) Prenatal magnetic resonance imaging assisting in differentiating between large degenerating intramural leiomyoma and complex adnexal mass during pregnancy. J Matern Fetal Med 9: 186-189.

11. Asuzu IM, Ugwa E, Ezike KN (2019) Hydropic degeneration of uterine leiomyoma mimicking a huge ovarian cyst. Int $\mathrm{J}$ Case Rep Images.

12. Sue W, Sarah SB (2009) Radiological appearances of uterine fibroids. Indian J Radiol Imaging 19: 222.

13. Ahmad S, Kovacs JE (2017) Complex cystic adnexal mass approximating the uterine myometrium. J Am Osteopath Coll Radiol.

14.(2007) American College of Obstetricians and Gynecologists. Practice bulletin no. 83: Management of adnexal masses, Washington. 